04,06

\title{
Электрическая поляризация неоднородно напряженных редкоземельных кристаллов со структурой граната и их пленок
}

\author{
() А.И. Попов ${ }^{1,2}$, Ч.К. Сабденов ${ }^{2,9}$ \\ ${ }^{1}$ Национальный исследовательский университет МИЭТ, \\ Москва, Россия \\ ${ }^{2}$ Московский фризико-технический институт, \\ Долгопрудный, Россия \\ I E-mail: sabdenovchingiz@gmail.com
}

Поступила в Редакцию 10 января 2019 г.

В окончательной редакции 10 января 2019 г.

Принята к публикации 15 января 2019 г.

Проанализировано влияние неоднородных деформаций на возникновение электрической поляризации редкоземельных соединений со структурой граната. Проведено исследование поляризации подсистемы редкоземельных ионов в областях неоднородных механических напряжений, порождаемых краевыми и винтовыми дислокациями. Изучено распределение электрической поляризации в неоднородно деформированных пленках редкоземельных гранатов.

Работа поддержана грантом РФФИ № 16-29-14005.

DOI: $10.21883 /$ FTT.2019.06.47682.351

\section{1. Введение}

В настоящее время проводятся интенсивные исследования физических свойств мультиферроиков, представляющих собой соединения, обладающие по крайней мере двумя из трех типов упорядочения: магнитного, электрического и упругого.

Главным образом изучаются мультиферроики у которых сосуществуют магнитное упорядочение и электрическая поляризация. В большинстве случаев подобные материалы представляют собой кристаллы с отсутствием центра инверсии $\left(\mathrm{BiFeO}_{3}\right.$, ферробораты и т.д.), что обеспечивает естественную возможность возникновения электрической поляризации, как спонтанной, так и индуцированной магнитным полем (магнитным порядком). В некоторых случаях появление электрической поляризации $P$ может происходить благодаря наличию у центросимметричных магнетиков пространственно нечетных структур [1,2]. С этой точки зрения к новому классу мультиферроиков можно отнести и кристаллы со структурой граната [2,3]. Так в „парамагнитном“ гранате $\mathrm{Mn}_{3} \mathrm{Al}_{2} \mathrm{Si}_{3} \mathrm{O}_{12}$ подсистема ионов $\mathrm{Mn}$ образует антисимметричную антиферромагнитную структуру, допускающую существование электрической поляризации [2]. В редкоземельных феррит-гранатах редкоземельные (Р3) ионы находятся под воздействием поля обменного $\mathrm{R}-\mathrm{Fe}-$ взаимодействия. При этом структуры магнитных моментов P3 ионов являются четными. Однородное магнитное поле обменного $\mathrm{R}$-Fe-взаимодействия индуцирует антисегнетоэлектрические структуры электрических дипольных моментов Р3 ионов с нулевой поляризацией [2]. Возникновение электродипольных моментов у Р3 ионов обусловлено отсутствием пространственной инверсии у окружения этих ионов в структуре граната.

Большой интерес для подобных соединений (центросимметричные среды, содержащие ионы, локальная симметрия окружения которых не содержит центра инверсии) представляют собой исследования их откликов на воздействия неоднородных полей. Так, в работах $[3,4]$ было показано, что в редкоземельных феррит-гранатах в областях неоднородного магнитного поля, в частности, в доменных границах возникает отличная от нуля поляризация подсистемы Р3 ионов.

Важно, что для указанных материалов возможно возникновение поляризации также и под воздействием неоднородных упругих напряжений (неоднородный пьезоэффект), о чем было указано в [5]. В данной работе была рассмотрена поляризация редкоземельных кристаллов гранатов, обусловленная неоднородными деформациями, возникающими при распространении упругих волн. Отметим, что в качестве поляризации в [3-5] был взят увеличенный в два раза электрический дипольный момент примитивной ячейки (отнесенный к ее объему), что является адекватным для однородных воздействий. Однако в случае неоднородных воздействий более корректным является использование элементарной ячейки. В настоящей работе исследована электрическая поляризация в кристаллах редкоземельных гранатов, возникающая вследствие неоднородных механических напряжений, порождаемых дислокациями, и в пленках редкоземельных гранатов, в которых неоднородные напряжения образуются в процессе их роста из-за неполного соответствия структур подложки и пленки. 
Таблица 1. Координаты $c$-позиций Р3 ионов в элементарной ячейке (в единицах ячейки)

\begin{tabular}{c|c|c|c|c|c|c|c|c|c|c|c|c}
\hline$k$ & 1 & 2 & 3 & 4 & 5 & 6 & 7 & 8 & 9 & 10 & 11 & 12 \\
\hline $\mathbf{r}^{(k)}$ & $0 \frac{3}{4} \frac{3}{8}$ & $0 \frac{1}{4} \frac{1}{8}$ & $\frac{3}{8} 0 \frac{3}{4}$ & $\frac{1}{8} 0 \frac{1}{4}$ & $\frac{3}{4} \frac{3}{8} 0$ & $\frac{1}{4} \frac{1}{8} 0$ & $0 \frac{1}{4} \frac{5}{8}$ & $0 \frac{3}{4} \frac{7}{8}$ & $\frac{5}{8} 0 \frac{1}{4}$ & $\frac{7}{8} 0 \frac{3}{4}$ & $\frac{1}{4} \frac{5}{8} 0$ & $\frac{3}{4} \frac{7}{8} 0$ \\
\hline \hline$k$ & 13 & 14 & 15 & 16 & 17 & 18 & 19 & 20 & 21 & 22 & 23 & 24 \\
\hline $\mathbf{r}^{(k)}$ & $\frac{1}{2} \frac{1}{4} \frac{7}{8}$ & $\frac{1}{2} \frac{3}{4} \frac{5}{8}$ & $\frac{7}{8} \frac{1}{2} \frac{1}{4}$ & $\frac{5}{8} \frac{1}{2} \frac{3}{4}$ & $\frac{1}{4} \frac{7}{8} \frac{1}{2}$ & $\frac{3}{4} \frac{5}{8} \frac{1}{2}$ & $\frac{1}{2} \frac{3}{4} \frac{1}{8}$ & $\frac{1}{2} \frac{1}{4} \frac{3}{8}$ & $\frac{1}{8} \frac{1}{2} \frac{3}{4}$ & $\frac{3}{8} \frac{1}{2} \frac{1}{4}$ & $\frac{3}{4} \frac{1}{8} \frac{1}{2}$ & $\frac{1}{4} \frac{3}{8} \frac{1}{2}$
\end{tabular}

\section{2. Кристаллическая структура редкоземельных гранатов}

Р3 соединения со структурой граната обладают рядом уникальных магнитных, магнитоупругих, магнитооптических и магнитоэлектрических свойств, обусловленных в большинстве случаев наличием в их составе P3 ионов [6-8]. Они имеют общую формулу $\mathrm{R}_{3} \mathrm{M}_{5} \mathrm{O}_{12}$, где $\mathrm{R}$ означает Р3 ион или ион иттрия $\mathrm{Y}^{3+}, \mathrm{M}-$ ион металла.

Важно, что структура граната допускает широкие возможности замещения одних ионов (как магнитных, так и немагнитных) на другие. Р3 гранаты являются кубическими магнетиками, обладающими весьма сложной кристаллографической структурой, описываемой пространственной группой $O_{h}^{10}-I a 3 d$. Элементарная ячейка, объемно-центрированная кубическая, включает в себя восемь формульных единиц $\mathrm{R}_{3} \mathrm{M}_{5} \mathrm{O}_{12}$, т. е. 160 атомов. Сторона элементарной ячейки имеет длину $\sim 12 \AA$. Важное значение для нас имеет тот факт, что Р3 ионы в кристаллах гранатов размещены по 6 кристаллографически неэквивалентным местам, обладающим более низкой, чем кубическая, симметрией окружения - додекаэдрическим местам или $c$-местам. Симметрия окружения $c$-мест в гранатах описывается точечной группой $D_{2}$, которая не содержит пространственной инверсии (что является принципиально важным обстоятельством для понимания магнитоэлектрики гранатов $[2,3])$. Элементарная ячейка кристалла граната состоит из двух примитивных ячеек. Расположение 12 Р3 ионов в первой примитивной ячейке приведено, например, в [9]. Остальные 12 Р3 ионов занимают позиции, образующиеся при трансляции на $\left[\frac{1}{2} \frac{1}{2} \frac{1}{2}\right]$ (в единицах ячейки) $c$-мест ионов первой ячейки. Координаты всех 24-х Р3 ионов в элементарной ячейке приведены в табл. 1.

Оси симметрии окружения $c$-мест:

$$
\begin{gathered}
\mathbf{e}_{x}^{(1,2)}=\left(\frac{1}{\sqrt{2}} \pm \frac{1}{\sqrt{2}} 0\right), \quad \mathbf{e}_{y}^{(1,2)}=\left(\mp \frac{1}{\sqrt{2}} \frac{1}{\sqrt{2}} 0\right), \\
\mathbf{e}_{z}^{(1,2)}=(001) ; \quad \mathbf{e}_{x}^{(3,4)}=\left(0 \frac{1}{\sqrt{2}} \pm \frac{1}{\sqrt{2}}\right), \\
\mathbf{e}_{y}^{(3,4)}=\left(0 \mp \frac{1}{\sqrt{2}} \frac{1}{\sqrt{2}}\right), \quad \mathbf{e}_{z}^{(3,4)}=(100) ; \\
\mathbf{e}_{x}^{(5,6)}=\left( \pm \frac{1}{\sqrt{2}} 0 \frac{1}{\sqrt{2}}\right), \quad \mathbf{e}_{y}^{(5,6)}=\left(\frac{1}{\sqrt{2}} 0 \mp \frac{1}{\sqrt{2}}\right), \\
\mathbf{e}_{z}^{(5,6)}=(010) ; \quad \mathbf{e}_{\alpha}^{(k+6)}=-\mathbf{e}_{\alpha}^{(k)}, \quad \alpha=x, y, z .
\end{gathered}
$$

Нумерация мест ионов в ячейке (табл. 1) выбрана такой, что окружение места $(k+6)(k=1 \ldots, 18)$ отличается от окружения $k$-го места операцией пространственной инверсии, что вытекает из соотношения $\mathbf{e}_{\alpha}^{(k+6)}=-\mathbf{e}_{\alpha}^{(k)}, \alpha=x, y, z$. Отсутствие пространственной инверсии окружения Р3 иона приводит к появлению электрического дипольного момента у иона при воздействии магнитного поля, либо механических напряжений $[2-5]$. В результате однородных воздействий (магнитное поле, механические напряжения) возникают антисегнетоэлектрические структуры электродипольных моментов с нулевым результирующим дипольных моментом ячейки $[2-5]$. Отличный от нуля электрический дипольный момент $\mathbf{P}$ ячейки может возникнуть в пленках гранатов вследствие влияния неоднородных механических напряжений, возникающих в процессе роста пленок Р3 гранатов, а также и в монокристаллах в области дислокаций.

\section{3. Механические напряжения и электрическая поляризация редкоземельных гранатов}

В работе [5] было показано, что в результате совместного воздействия деформации решетки кристалла граната и внешнего электрического поля $\mathbf{E}$ на спектр $k$-го Р3 иона возникают отличные от нуля линейные по величине напряженности поля $E$ поправки к уровням энергии иона, которые могут быть представлены эффективным оператором пьезоэлектрического взаимодействия (в локальных осях $k$-го иона)

$$
\begin{aligned}
& V^{(k)}=-A\left(E _ { x } ^ { ( k ) } \left[U_{y z}^{(k)}\left(C_{-2}^{2}+C_{2}^{2}-\sqrt{\frac{2}{3}} C_{0}^{2}\right)\right.\right. \\
& \left.+U_{x z}^{(k)} i\left(C_{-2}^{2}-C_{2}^{2}\right)+U_{x y}^{(k)}\left(C_{-1}^{2}-C_{1}^{2}\right)\right] \\
& +E_{y}^{(k)}\left[U_{y z}^{(k)} i\left(C_{-2}^{2}-C_{2}^{2}\right)-U_{x z}^{(k)}\left(C_{-2}^{2}+C_{2}^{2}+\sqrt{\frac{2}{3}} C_{0}^{2}\right)\right. \\
& \left.+U_{x y}^{(k)} i\left(C_{-1}^{2}+C_{1}^{2}\right)\right]+E_{z}^{(k)}\left[U_{y z}^{(k)}\left(C_{-1}^{2}-C_{1}^{2}\right)\right. \\
& \left.\left.+U_{x z}^{(k)} i\left(C_{-1}^{2}+C_{1}^{2}\right)+U_{x y}^{(k)} 2 \sqrt{\frac{2}{3}} C_{0}^{2}\right]\right),
\end{aligned}
$$

где $A-$ константа взаимодействия (см. [5]) $C_{q}^{p}=\sum_{i=1}^{N} C_{q}^{p}(i), C_{q}^{p}(i)$ - одноэлектронный неприводимый 
оператор, определяемый приведенным матричных элементом $\left\langle l^{\prime}\left\|C^{p}\right\| l\right\rangle=\sqrt{2 l+1} C_{l 0 p 0}^{l^{\prime} 0}, \quad l^{\prime} \quad$ и $\quad l \quad-$ орбитальные квантовые числа состояний электрона, $C_{a \alpha b \beta}^{c \gamma}-$ коэффициент Клебша-Гордана, $N-$ число $f$-электронов иона, $U_{\alpha \beta}^{(k)}-$ компоненты тензора деформации в системе координат $k$-го иона. Основным взаимодействием, формирующим спектр Р3 ионов в структуре граната, является воздействие кристаллического поля. Взаимодействие иона с магнитным полем (исключая сверхсильные поля $H>10^{2} \mathrm{~T}$ ), как и обменное взаимодействие с ионами железа (для феррит-гранатов) можно учесть в качестве возмущения.

При усреднении (1) по состояниям Р3 иона в кристаллическом поле граната отличными от нуля средними обладают только инвариантные относительно преобразований группы $D_{2}$ операторы $C_{0}^{2}$ и $C_{-2}^{2}+C_{2}^{2}$. В этом случае вклад пьезоэлектрического взаимодействия (1) $k$-го Р3 иона в свободную энергию граната

$$
\begin{aligned}
& W^{(k)}=-A\left\{E_{x}^{(k)} U_{y z}^{(k)}\left\langle C_{-2}^{2}+C_{2}^{2}-\sqrt{\frac{2}{3}} C_{0}^{2}\right\rangle\right. \\
& \left.-E_{y}^{(k)} U_{x z}^{(k)}\left\langle C_{-2}^{2}+C_{2}^{2}+\sqrt{\frac{2}{3}} C_{0}^{2}\right\rangle+E_{z}^{(k)} U_{x y}^{(k)} 2 \sqrt{\frac{2}{3}}\left\langle C_{0}^{2}\right\rangle\right\},
\end{aligned}
$$

где $\left\langle C_{q}^{p}\right\rangle=\sum_{n}\left\langle\Psi_{n}\left|C_{q}^{p}\right| \Psi_{n}\right\rangle \rho_{n}, \rho_{n}=e^{-E_{n} / k T}\left(\sum_{n} e^{-E_{n} / k T}\right)^{-1}$, $E_{n}, \Psi_{n}-$ уровни энергии и волновые функции Р3 иона в кристаллическом поле. Из (2) следует, что электрический дипольный момент $k$-го иона

$$
\begin{aligned}
& \mathbf{d}^{(k)}=-\frac{\partial W^{(k)}}{\partial \vec{E}}=A\left\{\mathbf{e}_{x}^{k} U_{y z}^{(k)}\left\langle C_{-2}^{2}+C_{2}^{2}-\sqrt{\frac{2}{3}} C_{0}^{2}\right\rangle\right. \\
& \left.-\mathbf{e}_{y}^{k} U_{x z}^{(k)}\left\langle C_{-2}^{2}+C_{2}^{2}+\sqrt{\frac{2}{3}} C_{0}^{2}\right\rangle+\mathbf{e}_{z}^{k} U_{x y}^{(k)} 2 \sqrt{\frac{2}{3}}\left\langle C_{0}^{2}\right\rangle\right\} .
\end{aligned}
$$

В кристаллографической система координат $X=[100]$, $Y=[010], Z=[001]$ компоненты тензора деформации $U_{\alpha \beta}^{(k)}$ для $k=1, \ldots, 6$ приведены в табл. 2.

При однородных деформациях электрические дипольные моменты Р3 ионов образуют сложные антисегнетоэлектрические структуры с нулевым результирующим моментом ячейки (поскольку $\left.\mathbf{p}^{(k+6)}=-\mathbf{p}^{(k)}[5]\right)$. В случае неоднородных деформаций возникает раскомпенса-

Таблица 2. Компоненты тензора деформации $U_{\alpha \beta}^{(k)}$ в кристаллографической системе координат

\begin{tabular}{c|c|c|c}
\hline$k$ & $U_{x y}^{(k)}$ & $U_{x z}^{(k)}$ & $U_{y z}^{(k)}$ \\
\hline $1 ; 2$ & $\mp\left(U_{X X}-U_{Y Y}\right) / 2$ & $\left(U_{X Z} \pm U_{Y Z}\right) / \sqrt{2}$ & $\left(U_{Y Z} \mp U_{X Z}\right) / \sqrt{2}$ \\
$3 ; 4$ & $\mp\left(U_{Y Y}-U_{Z Z}\right) / 2$ & $\left(U_{X Y} \pm U_{X Z}\right) / \sqrt{2}$ & $\left(U_{X Z} \mp U_{X Y}\right) / \sqrt{2}$ \\
$5 ; 6$ & $\mp\left(U_{Z Z}-U_{X X}\right) / 2$ & $\left(U_{Y Z} \pm U_{X Y}\right) / \sqrt{2}$ & $\left(U_{X Y} \mp U_{Y Z}\right) / \sqrt{2}$
\end{tabular}

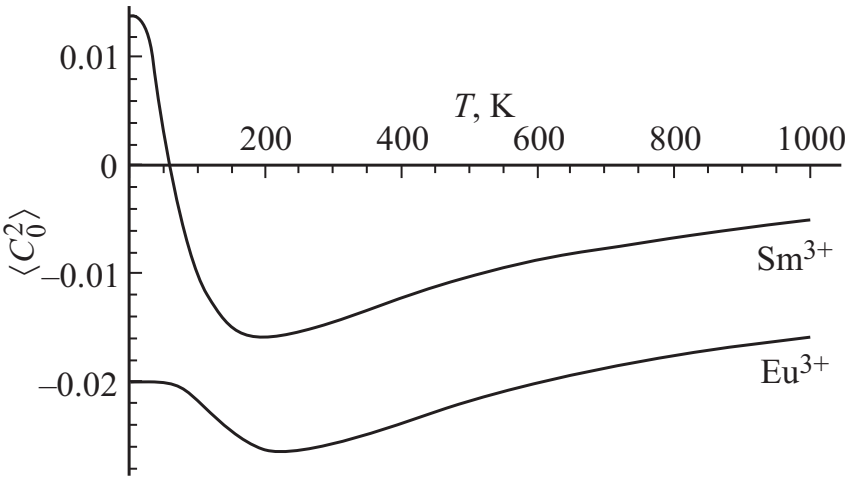

Рис. 1. Температурные зависимости $\left\langle C_{0}^{2}\right\rangle$ для $\mathrm{Eu}^{3+}$ и $\mathrm{Sm}^{3+}$.

ция электрических дипольных моментов Р3 ионов в ячейке, что приводит к возникновению отличного от нуля момента ячейки, а, следовательно, и к электрической поляризации материала. Для ее нахождения используем соотношения $U_{\alpha \beta}\left(\mathbf{r}_{k}\right)-U_{\alpha \beta}\left(\mathbf{r}_{k^{\prime}}\right)=\nabla U_{\alpha \beta}\left(\mathbf{r}_{k}-\mathbf{r}_{k^{\prime}}\right)$, просуммируем (3) по всем 24 ионам элементарной ячейки и получим ее пьезоэлектрическую энергию $W_{p e}=\sum_{k=1}^{24} W^{(k)}$. Электрическую поляризацию материала определим как дипольный момент ячейки, деленный на ее объем

$$
P_{\alpha}=-\frac{1}{a^{3}} \frac{\partial W_{p e}}{\partial E_{\alpha}}
$$

$\alpha=X, Y, Z, a \sim 12$ - размер ячейки.

В результате получим

$$
\begin{aligned}
& P_{X}=Q\left(\frac{\partial U_{X Y}}{\partial Y}-\frac{\partial U_{X Z}}{\partial Z}+\frac{\partial}{\partial X}\left(U_{Y Y}-U_{Z Z}\right)\right), \\
& P_{Y}=Q\left(\frac{\partial U_{Y Z}}{\partial Z}-\frac{\partial U_{X Y}}{\partial X}+\frac{\partial}{\partial Y}\left(U_{Z Z}-U_{X X}\right)\right), \\
& P_{Z}=Q\left(\frac{\partial U_{X Z}}{\partial X}-\frac{\partial U_{Y Z}}{\partial Y}+\frac{\partial}{\partial Z}\left(U_{X X}-U_{Y Y}\right)\right),
\end{aligned}
$$

$Q=\frac{A}{a^{2}} \sqrt{\frac{2}{3}}\left\langle C_{0}^{2}\right\rangle$. Температурная зависимость поляризации Р3 ионов (5), порождаемой неоднородными деформациями решетки, определяется зависимостью от температуры среднего значения оператора $C_{0}^{2}$, представляющего собой оператор $Q_{z z}$ квадрупольного момента $f$-оболочки Р3 иона. Отметим, что при $k T<\Delta E_{\text {cr }}$, где $\Delta E_{\mathrm{cr}}$ - расщепление основного мультиплета Р3 иона в кристаллическом поле, $\left\langle C_{0}^{2}\right\rangle$ в большинстве случаев слабо зависит от температуры. Для характерных значений $\Delta E_{\mathrm{cr}} \sim 10^{2}-10^{3} \mathrm{~cm}^{-1}[6]$ это область температур $T<10^{3} \mathrm{~K}$.

В состав редкоземельных гранатов часто входят ионы $\mathrm{Eu}^{3+}$ и $\mathrm{Sm}^{3+}$. Для расчета зависимости от температуры поляризации ионов самария воспользуемся данными о спектре этого иона в кристаллическом поле феррит- 
граната, приведенными в [10], а для европия — данными о параметрах кристаллического поля и обменного взаимодействия $[11,12]$. Отметим, что в [10] была использована система координат, в которой локальные оси $\mathbf{e}_{x}$ были ориентированы вдоль осей типа [100], а оси $\mathbf{e}_{z}-$ вдоль осей типа [110]. Зависимости $\left\langle C_{0}^{2}\right\rangle$ от температуры для ионов европия и самария, описывающие температурное поведение поляризации соответствующих соединений, приведены на рис. 1.

\section{4. Поляризация редкоземельных гранатов и пленок, порождаемая полем напряжений краевых и винтовых дислокаций и дислокаций несоответствия}

Структуры реальных кристаллов обладают рядом несовершенств, называемых дислокациями [13]. Основными видами дислокаций являются краевые и винтовые дислокации. Краевая дислокация представляет собой дефект кристаллической структуры, приводящий к деформации, вызванный появлением лишней атомной плоскости. Винтовую дислокацию можно представить себе, если мысленно сделать в кристалле разрез, а затем сдвинуть части кристалла по обе стороны разреза относительно друг друга на одно межатомное расстояние параллельно краю разреза. Дислокации порождают в кристалле неоднородные упругие напряжения. Так, поле напряжений краевой дислокации определяется выражениями [13]

$$
\begin{gathered}
\sigma_{x x}=-G b \frac{y\left(3 x^{2}+y^{2}\right)}{2 \pi(1-\mu)\left(x^{2}+y^{2}\right)^{2}}, \\
\sigma_{y y}=G b \frac{y\left(x^{2}-y^{2}\right)}{2 \pi(1-\mu)\left(x^{2}+y^{2}\right)^{2}}, \\
\sigma_{x y}=G b \frac{x\left(x^{2}-y^{2}\right)}{2 \pi(1-\mu)\left(x^{2}+y^{2}\right)^{2}} .
\end{gathered}
$$

Поле напряжений винтовой дислокации [13]

$$
\begin{gathered}
\sigma_{x z}=\sigma_{z x}=-\frac{G b y}{2 \pi\left(x^{2}+y^{2}\right)}, \\
\sigma_{z y}=\sigma_{y z}=\frac{G b x}{2 \pi\left(x^{2}+y^{2}\right)} .
\end{gathered}
$$

В (6) и (7) ось $z \|$ линии дислокации, $G$ - модуль упругости при сдвиге, $b \sim a-$ длина вектора Бюргерса, $\mu$ - коэффициент Пуассона. Формулы (6) и (7) справедливы вне ядра дислокации - области радиуса не более 1.5-2 векторов Бюргерса. Для кубического кристалла компоненты тензоров напряжения и деформации связаны соотношениями [14]

$$
\begin{gathered}
U_{\alpha \beta}=\frac{1}{2 c_{44}} \sigma_{\alpha \beta}, \quad(\alpha \neq \beta), \\
U_{\alpha \alpha}-U_{\beta \beta}=\frac{\sigma_{\alpha \alpha}-\sigma_{\beta \beta}}{c_{11}-c_{12}},
\end{gathered}
$$

где $c_{n n}$ - коэффициенты упругости кубических кристаллов [14].

Пренебрежем для простоты различием упругих констант $2 c_{44}$ и $c_{11}-c_{12}$ (приближение изотропной упруго среды) и обозначим $2 c_{44}=c_{11}-c_{12} \equiv c$. Определим распределение поляризации, порождаемой краевой и винтовой дислокациями.

1. Краевая дислокация. Пусть линия краевой дислокации направлена вдоль оси [001]. Используем формулы (6) и (8) и найдем, исходя из (15)

$$
\begin{gathered}
P_{X}=4 q \frac{X Y\left(Y^{2}-X^{2}\right)}{\left(X^{2}+Y^{2}\right)^{3}}=-q \frac{\sin (2 \phi)}{\rho^{2}}, \\
P_{Y}=4 q \frac{X^{2}\left(X^{2}-3 Y^{2}\right)}{\left(X^{2}+Y^{2}\right)^{3}}=4 q \frac{\cos (3 \phi) \cos (\phi)}{\rho^{2}}, \\
P_{Z}=0,
\end{gathered}
$$

где $q=\frac{G b Q}{2 \pi(1-\mu) c}, X=\rho \cos (\phi), Y=\rho \sin (\phi)$.

2. Винтовая дислокация. В этом случае из (7), (8) и (5) найдем

$$
\begin{gathered}
P_{X}=P_{Y}=0, \\
P_{Z}=4 q(1-\mu) \frac{X Y}{\left(X^{2}+Y^{2}\right)^{2}}=2 q(1-\mu) \frac{\sin (2 \phi)}{\rho^{2}} .
\end{gathered}
$$

Из (9) и (10) вытекает, что краевые дислокации порождают поляризацию, ориентированную в плоскости, перпендикулярной линии дислокации, в то время как вектор поляризации, порождаемой винтовой дислокацией, направлен вдоль линии дислокации.

Неоднородные механические напряжения могут реализовываться в пленках Р3 гранатов в процессе их роста вследствие не полного согласования структур подложки и пленки. В начале роста пленки она находится в так называемом псевдоморфном состоянии, представляющем собой кристаллографическое продолжение решетки подложки. В этом состоянии пленка однородно напряжена и в ней возникает антисегнетоэлектрическая структура электродипольных моментов Р3 ионов с нулевой поляризацией. По мере роста пленки данное состояние становится энергетически невыгодным и в некотором слое, прилегающем к подложке, происходит релаксация напряжений несоответствия. В результате возникают семейства дислокаций несоответствия, которые приближенно можно представить в виде ряда краевых дислокаций, вызывающих неоднородные механические напряжения слоя пленки, прилегающего к подложке [15]. Рассмотрим пленку (001). В этом случае семейство краевых дислокаций (моделирующих дислокации несоответствия, линии которых параллельны осям [100] 

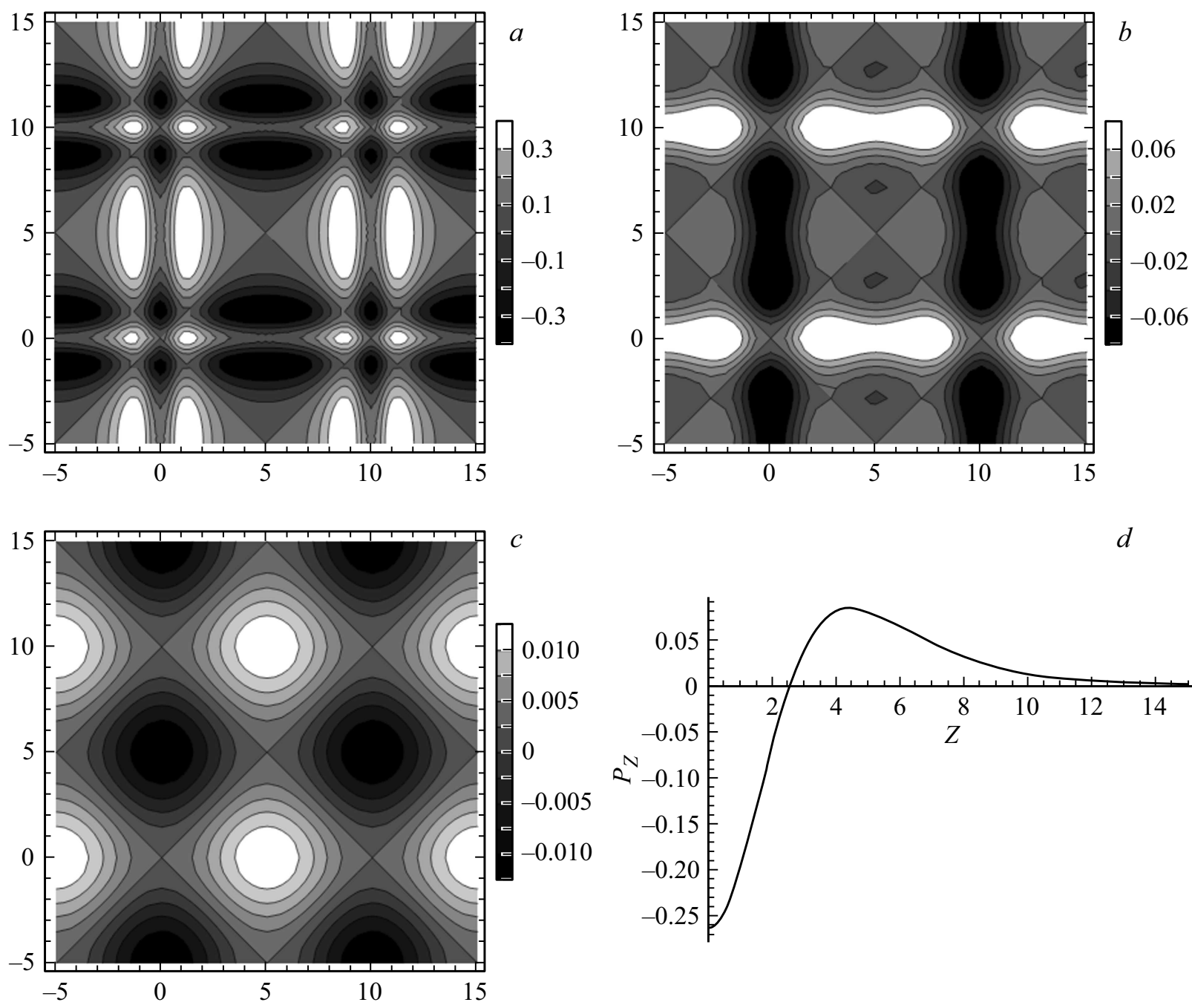

$d$

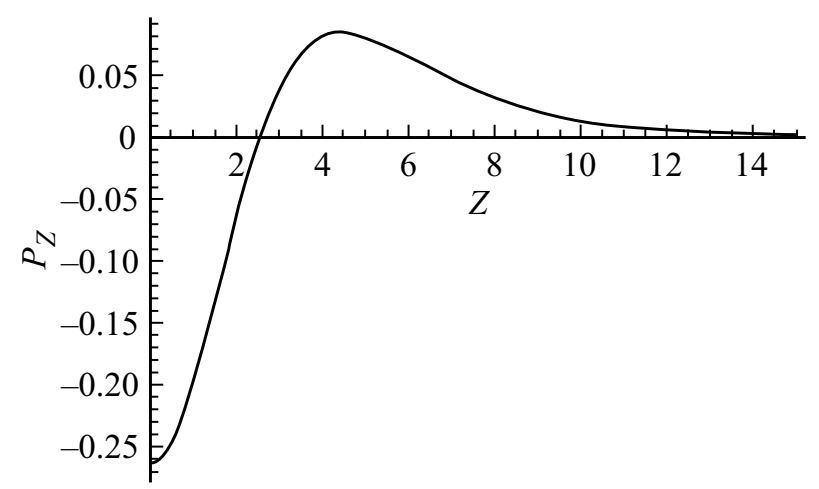

Рис. 2. Распределение компоненты поляризации $P_{Z}$ в плоскости пленки $(X, Y)$ при различных $Z: a-Z=2, b-Z=4, c-$ $Z=10 . d-$ зависимость $P_{Z}$ от глубины для $(X, Y)=(5,0)$.

и [010]), порождает неоднородные напряжения, которые определяются формулами

$$
\begin{gathered}
\sigma_{X X}(X, Z)=\sum_{n} \sigma_{X X}^{(n)}(X, Z), \quad \sigma_{Y Y}(Y, Z)=\sigma_{X X}(Y, Z), \\
\sigma_{Z Z}(X, Y, Z)=\sum_{n}\left(\sigma_{Z Z}^{(n)}(X, Z)+\sigma_{Z Z}^{(n)}(Y, Z)\right), \\
\sigma_{X Z}(X, Z)=\sum_{n} \sigma_{X Z}^{(n)}(X, Z), \quad \sigma_{Y Z}(Y, Z)=\sigma_{X Z}(Y, Z),
\end{gathered}
$$

где

$$
\begin{gathered}
\sigma_{X X}^{(n)}(\xi, Z)=-S \frac{Z\left[3(\xi-n d)^{2}+Z^{2}\right]}{\phi^{(n)}(\xi, Z)}, \\
\sigma_{Z Z}^{(n)}(\xi, Z)=S \frac{Z\left[(\xi-n d)^{2}-Z^{2}\right]}{\phi^{(n)}(\xi, Z)}, \\
\sigma_{X Z}^{(n)}(\xi, Z)=S \frac{(\xi-n d)\left[(\xi-n d)^{2}-Z^{2}\right]}{\phi^{(n)}(\xi, Z)},
\end{gathered}
$$

$$
\begin{gathered}
\phi^{(n)}(\xi, Z)=\left[(\xi-n d)^{2}+Z^{2}\right]^{2} \\
\xi=X, Y ; \quad S=\frac{G b}{2 \pi(1-\mu)} .
\end{gathered}
$$

В (11), (12) $n=0, \pm 1, \pm 2, \ldots, \pm N(2 N+1-$ число дислокаций $), d-$ период дислокаций. Проведем $d$ (11) суммирование по $n$, полагая $N=\infty$ и, используя равенство

$$
\pi \cot (\pi x)=\lim _{N \rightarrow \infty} \sum_{n=-N}^{N} \frac{1}{x+n}
$$

получим

$$
\begin{aligned}
\sigma_{X X}= & \sum_{n} \sigma_{X X}^{(n)}(X, Z)=\frac{2 \pi^{2} S Z}{d^{2}} \frac{\cos \left(\frac{2 \pi X}{d}\right) \cosh \left(\frac{2 \pi Z}{d}\right)-1}{\left(\cosh \left(\frac{2 \pi Z}{d}\right)-\cos \left(\frac{2 \pi X}{d}\right)\right)^{2}} \\
& -\frac{2 \pi S / d}{\operatorname{coth}\left(\frac{\pi Z}{d}\right) \sin ^{2}\left(\frac{\pi X}{d}\right)+\tanh \left(\frac{\pi Z}{d}\right) \cos ^{2}\left(\frac{\pi X}{d}\right)},
\end{aligned}
$$




$$
\begin{gathered}
\sigma_{Z Z}=\sum_{n}\left(\sigma_{Z Z}^{(n)}(X, Z)+\sigma_{Z Z}^{(n)}(Y, Z)\right)=\frac{2 \pi^{2} S Z}{d^{2}} \\
\times\left(\frac{1-\cos \left(\frac{2 \pi X}{d}\right) \cosh \left(\frac{2 \pi Z}{d}\right)}{\left(\cosh \left(\frac{2 \pi Z}{d}\right)-\cos \left(\frac{2 \pi X}{d}\right)\right)^{2}}+\frac{1-\cos \left(\frac{2 \pi Y}{d}\right) \cosh \left(\frac{2 \pi Z}{d}\right)}{\left(\cosh \left(\frac{2 \pi Z}{d}\right)-\cos \left(\frac{2 \pi Y}{d}\right)\right)^{2}}\right) \\
\sigma_{X Z}=\sum_{n} \sigma_{X Z}^{(n)}(X, Z) \\
=\frac{\pi S / d}{\cot \left(\frac{\pi X}{d}\right) \sinh ^{2}\left(\frac{\pi Z}{d}\right)+\tan \left(\frac{\pi X}{d}\right) \cosh ^{2}\left(\frac{\pi Z}{d}\right)} \\
\quad-\frac{2 \pi^{2} S Z}{d^{2}} \frac{\sin \left(\frac{2 \pi X}{d}\right) \sinh \left(\frac{2 \pi Z}{d}\right)}{\left(\cosh \left(\frac{2 \pi Z}{d}\right)-\cos \left(\frac{2 \pi X}{d}\right)\right)^{2}} .
\end{gathered}
$$

На рис. 2 визуализированы результаты, полученные из формул (5), (8)-(13). Для простоты опущены точные значения констант - полагается, что координаты и расстояние между линиями дислокаций отмечаются в единицах постоянной решетки, а поляризация в единицах $q$. Также полагается, что $d=10$.

\section{5. Заключение}

В настоящей работе исследовано возникновение электрической поляризации редкоземельных соединений со структурой граната, порождаемой неоднородными деформациями. Явление представляет собой неоднородный пьезоэффект в центросимметричных средах, в структуpax которых имеются ионы, симметрия окружения которых не содержит центра инверсии, и определяется не точечной, а пространственной симметрией кристаллов.

Изучена электрическая поляризация кристаллов редкоземельных гранатов, обладающих несовершенствами структуры, представляющими собой краевые и винтовые дислокации. Установлено, что поле неоднородных механических напряжений дислокаций порождает в кристалле поляризацию, спадающую по закону $\rho^{-2}$, где $\rho$ - расстояние от линии дислокации, и обладающую сложной угловой зависимостью. Причем в случае краевой дислокации вектор поляризации ориентирован в плоскости, перпендикулярной линии дислокации, а при винтовой дислокации поляризация направлена вдоль линии дислокации.

Исследована поляризация неоднородно напряженных пленок редкоземельных гранатов. Показано, что поле электрической поляризации пленок, порождаемое дислокациями несоответствия, имеет сложный вид периодической структуры, период которой определяется параметром несоответствия кристаллических структур подложки и пленки. Отметим также, что неоднородные упругие напряжения могут создаваться специально путем ионной бомбардировки поверхности пленки.

\section{Список литературы}

[1] A.K. Zvezdin, A.A. Mukhin. JETP Lett. 88, 505 (2008).

[2] A.I. Popov, D.I. Plokhov, A.K. Zvezdin. Phys. Rev. B 90, 214427 (2014).

[3] A.I. Popov, Z.V. Gareeva, A.K. Zvezdin. Phys. Rev. B 92, 144420 (2015).

[4] A.I. Popov, K.A. Zvezdin, Z.V. Gareeva, F.A. Mazhitova, R.M. Vakhitov, A.R. Yumaguzin, A.K. Zvezdin. J. Phys.: Cond. Matter 28, 456004 (2016).

[5] А.И. Попов, Ч.К. Сабденов, К.А. Звездин. ФТТ 59, 2252 (2017).

[6] А.К. Звездин, В.М. Матвеев, А.А. Мухин, А.И. Попов. Редкоземельные ионы в магнитоупорядоченных кристаллах. Наука, М. (1985). 296 с.

[7] A.K. Zvezdin, V.A. Kotov. Modern magnetooptics and magnetooptical materials. CRC Press (1997). 404 c.

[8] К.П. Белов, Н.А. Белянчикова, Р.З. Левитин, С.А. Никитин. Редкоземельные ферро- и антиферромагнетики. Наука, М. (1965). 449 c.

[9] Ю.А. Изюмов, Р.П. Озеров, В.Е. Найш. Нейтронография а магнетиков. Атомиздат, М. (1981). 311 с.

[10] О.А. Дорофеев, А.И. Попов. ФТТ 31, 124 (1989).

[11] А.К. Звездин, Р.3. Левитин, А.С. Маркосян, А.А. Мухин, А.И. Попов. ФТТ 18, 387 (1976).

[12] M.E. Foglio, J.H. Van Vleck. Proc. R. Soc. Lond. A 336, 115 (1974).

[13] D. Hull, D. Bacon Jr. Introduction to dislocations. Butterworth-Heinemann (2001). $242 \mathrm{c}$.

[14] Л.Д. Ландау, Е.М. Лифшиц. Теоретическая физика. Наука, М. (1989). T. 7. 264 c.

[15] Е.М. Труханов, А.В. Колесников, И.Д. Лошкарев. Фазовые переходы, межфазные границы и наноматериалы 1, 26 (2015).

Редактор Т.Н. Василевская 